\title{
Originalien
}

Ophthalmologe 2021 118 (Suppl 2):S166-S175 https://doi.org/10.1007/s00347-021-01411-7 Accepted: 27 April 2021

Published online: 4 June 2021

(c) Springer Medizin Verlag $\mathrm{GmbH}$, ein Teil von Springer Nature 2021

\author{
Lars-Olof Hattenbach ${ }^{1,2} \cdot$ Peter Heinz ${ }^{1,3} \cdot$ Nicolas Feltgen ${ }^{4} \cdot$ Hans Hoerauf $^{1,4}$. \\ Thomas Kohnen ${ }^{1,5} \cdot$ Siegfried Priglinger ${ }^{1,6} \cdot$ Werner Bachmann ${ }^{1,7}$. \\ Johannes Rieks ${ }^{1,8} \cdot$ Nicole Eter ${ }^{9}$. Thomas Reinhard ${ }^{1,10}$ \\ ${ }^{1}$ Commission for Cross-Sectoral Ophthalmology of the DOG (German Ophthalmological Society e. V.) and \\ BVA (Professional Association of German Ophthalmologists), Munich/Düsseldorf, Germany \\ ${ }^{2}$ Eye Clinic, Ludwigshafen Hospital, Ludwigshafen, Germany \\ ${ }^{3}$ Ophthalmology practice, Dr. Peter Heinz, Schlüsselfeld, Germany \\ ${ }^{4}$ Eye Clinic, University Hospital Göttingen, Göttingen, Germany \\ ${ }^{5}$ Eye Clinic, University Hospital Frankfurt/Main, Frankfurt/Main, Germany \\ ${ }^{6}$ Eye Clinic, Ludwig Maximilian University Munich, Munich, Germany \\ ${ }^{7}$ ReVis Eye Clinic Aschaffenburg, Aschaffenburg, Germany \\ ${ }^{8}$ Aurich Ophthalmological Care Center, Aurich, Germany \\ ${ }^{9}$ University Eye Hospital Münster, Münster, Germany \\ ${ }^{10}$ Department of Ophthalmology, University Hospital Freiburg, Freiburg, Germany
}

\section{Impact of the SARS-CoV-2 pandemic on ophthalmic care in Germany}

\section{Background}

In the first months of 2020, the SARSCoV-2 (severe acute respiratory syndrome coronavirus-2) pandemic led to massive restrictions in medical care worldwide and a drastic reduction in non-urgent outpatient and inpatient treatment [1-12]. Ophthalmology, a specialty with a high proportion of outpatient and planned surgical interventions for non-life-threatening indications, was particularly affected. The rapidly implemented conversion of hospitals in the early months of the pandemic to deal with the anticipated care of coronavirus disease 2019 (COVID-19) patients by providing the largest possible intensive care capacities, combined with considerable restrictions on access to hospitals and practices, greatly reduced and delayed patient admissions, the observance of hygiene and social-distancing rules and consequent longer waiting times, the ban on access to assistive accompanying persons, and, last but not least, the fear held by many patients of infection during a stay in a medical setting resulted in a clear decline in the number of patients in hospital outpatient departments and practices $[1,13,14]$.

A recent paper on the crisis strategies implemented by hospitals during the pandemic reported that emergencies and urgent referrals were reduced to as low as $30 \%$ of the usual number of cases after the treatment of ophthalmic patients was largely limited to urgent care in March and April [1]. However, no reliable figures on case numbers or shifts in the treatment spectrum have been available to date. It has also not yet been possible to assess the different effects of the pandemic on the various areas of ophthalmology, the extent to which outpatient and inpatient care structures were affected, or whether there were changes in the treatment of emergencies. Similarly, data were not available on the question of which factors led to a reduction in ophthalmic care and whether these factors had different effects on the various types of ophthalmic facilities. The present article, which is based on a survey conducted by the commission for cross-sectoral ophthalmology (Kommission Sektorenübergreifende Augenheilkunde), a joint commission of the German Ophthalmological Society
The German version of this article can be found under https://doi.org/10.1007/s00347020-01220-4 
Table 1 Number of participants in the survey on the impact of the SARS-CoV-2 pandemic by federal state

\begin{tabular}{|l|l|l|}
\hline Baden-Württemberg & 137 & $11.5 \%$ \\
\hline Bavaria & 191 & $16.1 \%$ \\
\hline Berlin & 53 & $4.5 \%$ \\
\hline Brandenburg & 34 & $2.9 \%$ \\
\hline Bremen & 14 & $1.2 \%$ \\
\hline Hamburg & 38 & $3.2 \%$ \\
\hline Hesse & 90 & $7.6 \%$ \\
\hline Mecklenburg-Western Pomerania & 23 & $1.9 \%$ \\
\hline Lower Saxony & 99 & $8.3 \%$ \\
\hline North Rhine-Westphalia & 263 & $22.1 \%$ \\
\hline Rhineland-Palatinate & 54 & $4.5 \%$ \\
\hline Saarland & 17 & $1.4 \%$ \\
\hline Saxony & 62 & $5.2 \%$ \\
\hline Saxony-Anhalt & 33 & $2.8 \%$ \\
\hline Schleswig-Holstein & 46 & $3.9 \%$ \\
\hline Thuringia & 25 & $2.1 \%$ \\
\hline Not specified & 11 & $0.9 \%$ \\
\hline
\end{tabular}

(DOG) and the Professional Association of German Ophthalmologists (BVA), is intended to provide the most comprehensive overview possible of the effects of the SARS-CoV-2 pandemic on ophthalmic care in Germany.

\section{Materials and methods}

The questions were distributed by means of an online survey conducted by the DOG of all 7291 working members of the DOG and BVA, totaling countrywide 7866 ophthalmologists employed predominantly in the outpatient sector, as well as in 103 university or municipal hospital departments of ophthalmology.

Questions and answers were previously agreed upon by the members of the commission on cross-sectoral ophthalmology, as well as the DOG and BVA. The survey was conducted anonymously. All respondents were asked to provide one response only from each eye center.

The options for specifying the type of facility included the differentiation into "maximum care provider," "surgical/ conservative care," or "conservative care," as well as "clinic" (i.e., hospital departments with inpatient beds included in the state bed plan), "medical care center (MCC)/group practice," "inpatient ward," and "private practice." Respondents were also able to indicate "other."
Questions on the restriction of ophthalmic care during the pandemic were related to the period from March 15 to April 15, 2020. To this end, participants were asked to what extent it was still possible to maintain outpatient or consulting hours, whereby it was possible to distinguish between "unrestricted," "reduced care," and "emergency care only," as well as "reduced intensity of treatment per case." The latter response option was intended to record qualitative restrictions in addition to quantitative restrictions on care, e.g., in cases where only a telephone consultation was possible.

In order to measure the reduction in conservative care compared to normal activity, information was requested on the percentage reduction or the number of cases compared to the period from January 15 to February 15, 2020. For information on restrictions in outpatient and inpatient surgeries, it was possible to specify the categories "unrestricted," "reduced care," and "emergency care only." In addition, information on the percentage reduction or the reduction in the number of cases compared to the period January 15 to February 15, 2020, was requested.

In order to record changes in the treatment of emergencies during the pandemic, case numbers for specific disorders during the period from March 15 to April 15, 2020, compared to the period from January 15 to February 15, 2020, were surveyed. These included the following diagnoses: retinal detachment, endophthalmitis, perforating eyeball injury, acute glaucoma, central retinal artery occlusion (CRAO), and anterior ischemic optic neuropathy (AION). Case numbers for keratoplasty and amniotic membrane transplantation were also surveyed.

The following response options were available to state the reasons that had led to a reduction in care during this period: legal, institutional, or professional policy requirements; operational/ physical restrictions; avoidance of risk to own health (e.g., due to a lack of protective equipment); or appointment cancellations by patients. In addition, questions were asked about staff absences due to SARS-CoV-2 infections or quarantine measures. Participants from institutions with inpatient beds were also asked to indicate the extent to which there had been a reduction in the number of beds and the deployment of personnel to other departments. Quantitative information was provided by the participating institution and expressed as percentages or absolute numbers. In the absence of percentages, absolute figures were converted into percentages in order to perform an overall comparison. Where numerical values were required for the analysis of specific questions, only answers with complete datasets were considered.

\section{Results}

A total of 1190 completed questionnaires were returned. The numbers of participants from the individual federal states are shown in - Table 1. Quantitative information was generally provided as percentage values. A total of 205 complete datasets were used for subgroup analyses of questions for which absolute numerical values were required.

Regarding the type of facility, 148 (12.4\%) reported being a maximum care provider, $485(40.8 \%)$ provided surgical and conservative care, and 556 (46.7\%) conservative care. In one case $(0.1 \%)$, no information was given in this regard. A total of 149 (12.5\%) of the participants 


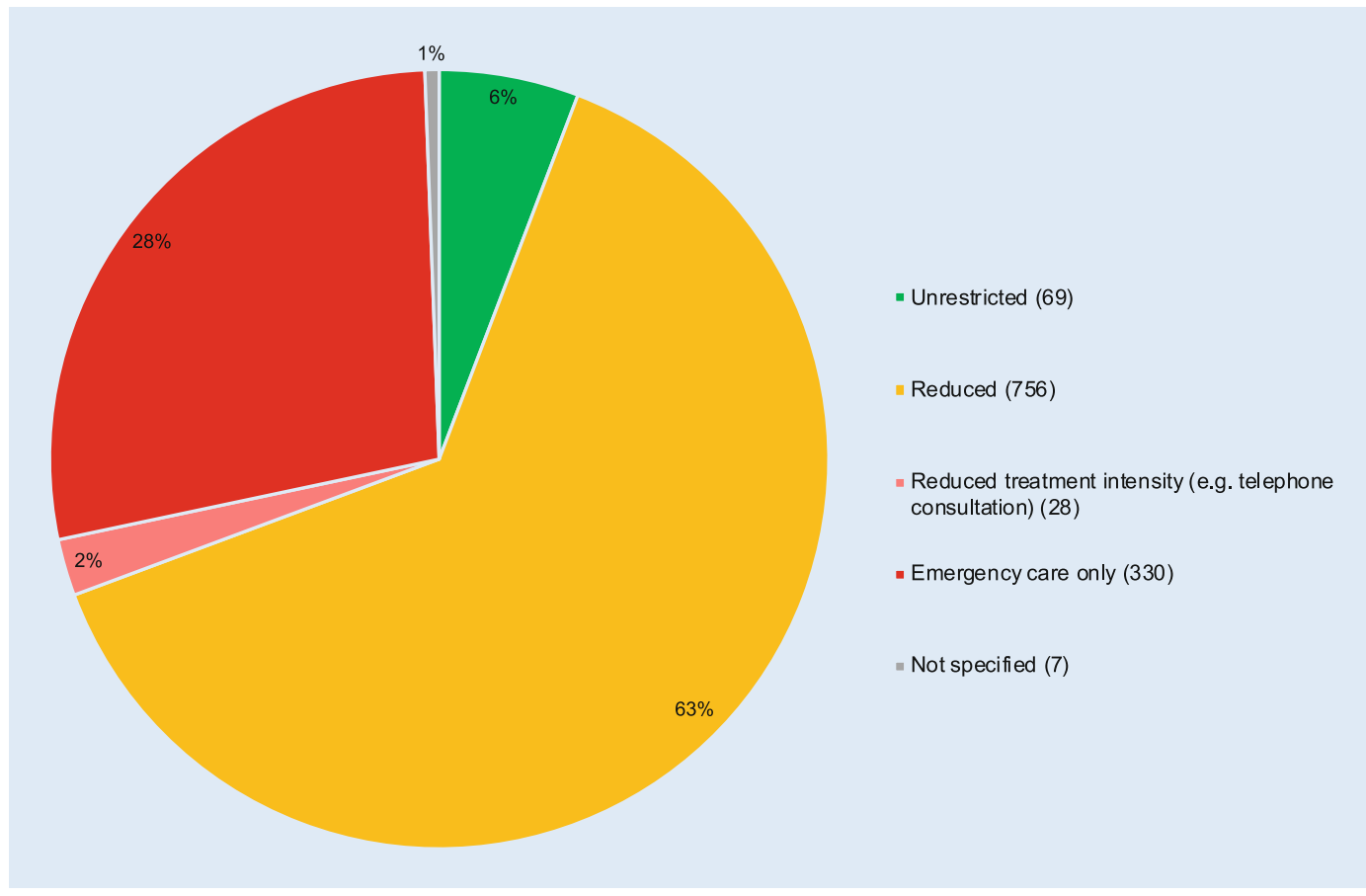

Fig. $1<$ Outpatient and practice-based consultations of all participants from March 15, 2020 to April 15, $2020(n=1190)$. Of a total of $91.0 \%, 63.0 \%$ provided reduced care and $28.0 \%$ emergency care only. Only $8.0 \%$ stated that unrestricted care had been available contacted gave the designation clinic, $493(41.4 \%)$ stated that they were run as an MCC or group practice, $16(1.3 \%)$ as an external physician with inpatient beds, $509(42.8 \%)$ as a private practice, and $21(1.8 \%)$ as "other" (including, e.g., eye bank, Medical Specialist Center of the German Armed Forces, nursing home care, etc.).

\section{Outpatient and consultation activities}

Of a total of 1190 participants surveyed, $69(5.8 \%)$ stated that they had carried out their outpatient clinic or consultation activities without restriction in the period from March 15 to April 15, 2020. A total of $756(63.5 \%)$ participants responded that they had maintained reduced care during this period, while 330 (27.7\%) had maintained emergency care only; $28(2.4 \%)$ of the respondents reported a reduction in the intensity of treatment per case, e.g., by telephone consultations; in seven cases $(0.6 \%)$, this question was not answered (• Fig. 1).

The extent to which conservative activity was restricted in relation to the type of institution is shown in - Fig. 2 $(n=1008)$. This shows that conservative care of patients was reduced or limited to the treatment of emergencies in
89.0-98.0\% of cases, regardless of the type of institution. Unrestricted outpatient or practice-based consultations was provided in $2.0 \%$ (clinics) to $9.0 \%$ (private practices providing conservative care) of all institutions. Significant differences were found in the restrictions to the emergency-only care of patients requiring conservative treatment, which was the case in $49.0 \%$ of clinics, only $8.0 \%$ of inpatient wards, $20.0-32.0 \%$ of surgical MCCs and group practices, and $26.0-29.0 \%$ of individual practices. Overall, based on an intact intersectoral cooperation, regular ophthalmic care for all cases requiring medical attention was maintained throughout the SARS-CoV-2 pandemic. Primary outpatient care was continuously provided by ophthalmic practices, and emergency care requiring conservative or surgical inpatient treatment was always ensured.

\section{Surgical activity}

A total of 467 datasets on surgical activities were evaluated, 85 of which were for individual practices, 257 for MCCs or group practices, 12 for inpatient wards, 105 for clinics, and eight for other facilities. On average, a reduction of $63.4 \%$ was reported for surgical activity, with inpatient wards reporting the highest at
$67.0 \%$, and MCCs and joint practices reporting on average the lowest value at $61.9 \%$. The extent of the reduction in surgical capacity is shown in $\mathbf{0}$ Fig. 3 . In $60.0 \%$ of all cases, there was a reduction in surgical capacity of $60.0 \%$ to $100.0 \%$. Only $6.0 \%$ reported a reduction of less than $20.0 \%$. - Fig. 4 shows the extent of restriction in outpatient and inpatient surgical activities in relation to the type of facility. According to this, the performance of inpatient procedures during the pandemic was limited to emergency care only in $75.0 \%$ of inpatient wards and in $71.0 \%$ of clinics. Furthermore, in $68.0 \%$ of clinics and in $42.0 \%$ of inpatient wards, outpatient surgery was also restricted to emergency surgery, while this was the case in $45 \%$ of surgical MCCs and group practices and $0 \%$ in private surgical practices. Unrestricted surgical care was exclusively provided on an outpatient basis and limited to $8 \%$ of private practices, $6 \%$ of MCCs and group practices, and $8 \%$ of inpatient wards.

An analysis of available complete datasets $(n=205)$ with case number values for surgical activities for the period from March 15, 2020, to April 15, 2020 , compared to the period from January 15 , 2020 , to February 15,2020 , revealed that the relative number of reduced surgeries in relation to the size of the institution 


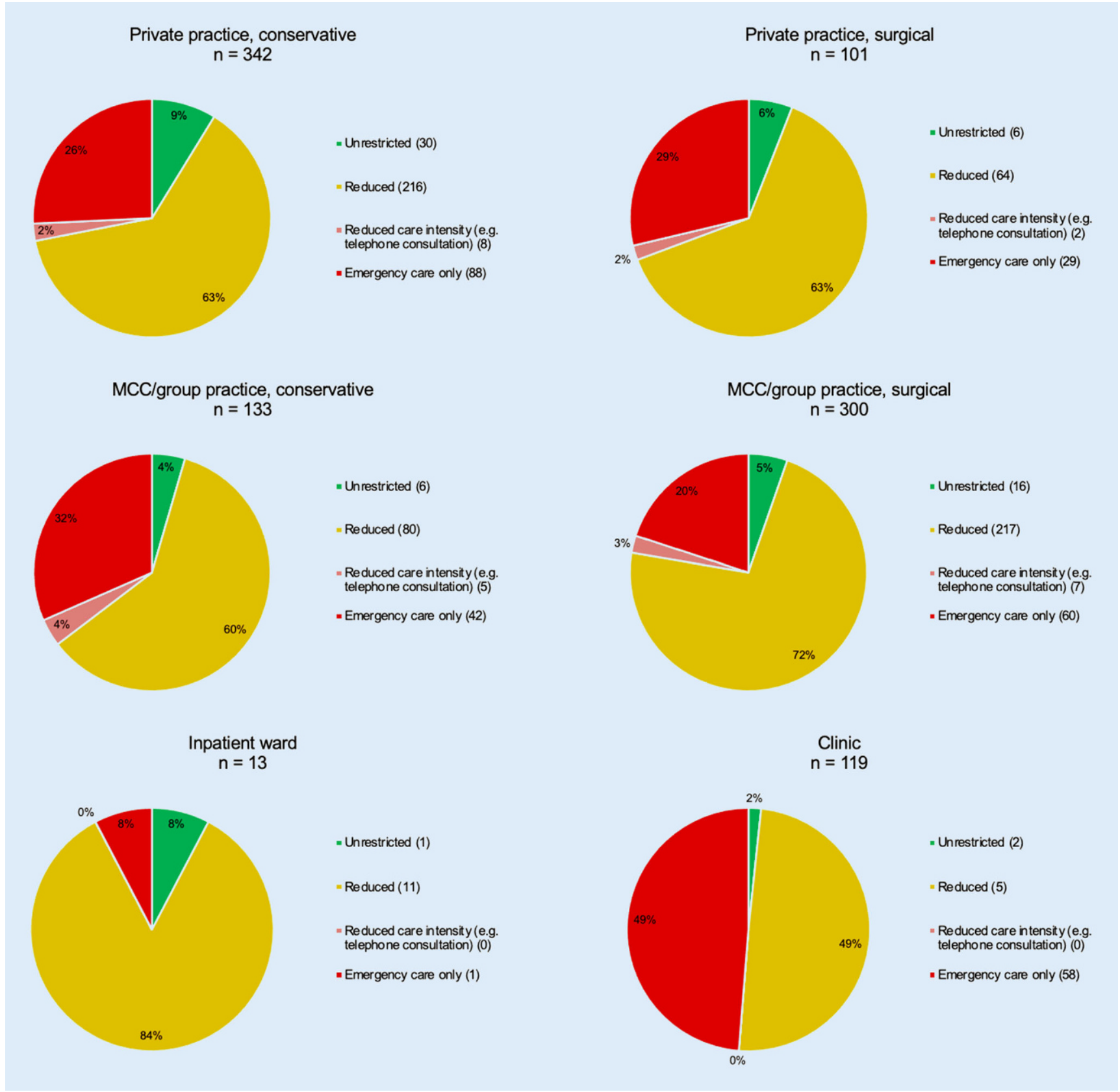

Fig. $2 \Delta$ Extent of restriction of conservative patients care in relation to the type of institution $(n=1008)$. Regardless of the type of facility, $89.0-98.0 \%$ offered reduced or limited emergency care. The latter, however, showed considerable variability with a share of $49.0 \%$ in clinics, $20-32 \%$ in surgical and conservative medical care centers (MCC), $26-29 \%$ in conservative or surgical private practices, and $8.0 \%$ in inpatient wards

was highest in clinics, followed by inpatient wards, surgical MCCs and group practices, as well as private surgical practices (• Fig. 5). The relative decrease in the number of surgeries was $60.0 \%$ on average, with a minimum of $50.1 \%$ (inpatient wards) and a maximum of $62.1 \%$ (MCCs and group practices).

\section{Emergencies and urgent procedures}

An analysis of the case numbers for retinal detachments, endophthalmitis, perforating eyeball injuries, acute glaucoma, CRAO and AION, as well as procedure numbers for penetrating keratoplasty and amniotic membrane transplantation from January 15 to February 15, 2020, and March 15 to April 15, 2020, with a total number of 2526 vs. 3804 cases, showed a significant decrease of $34.0 \%$ in the number of treated emergencies and urgent indications.

A comparison with regard to the type of disorder or indication is shown in - Fig. 6. A decrease in the number of emergencies was observed for the diagnoses retinal detachment (748 vs. 1148) 


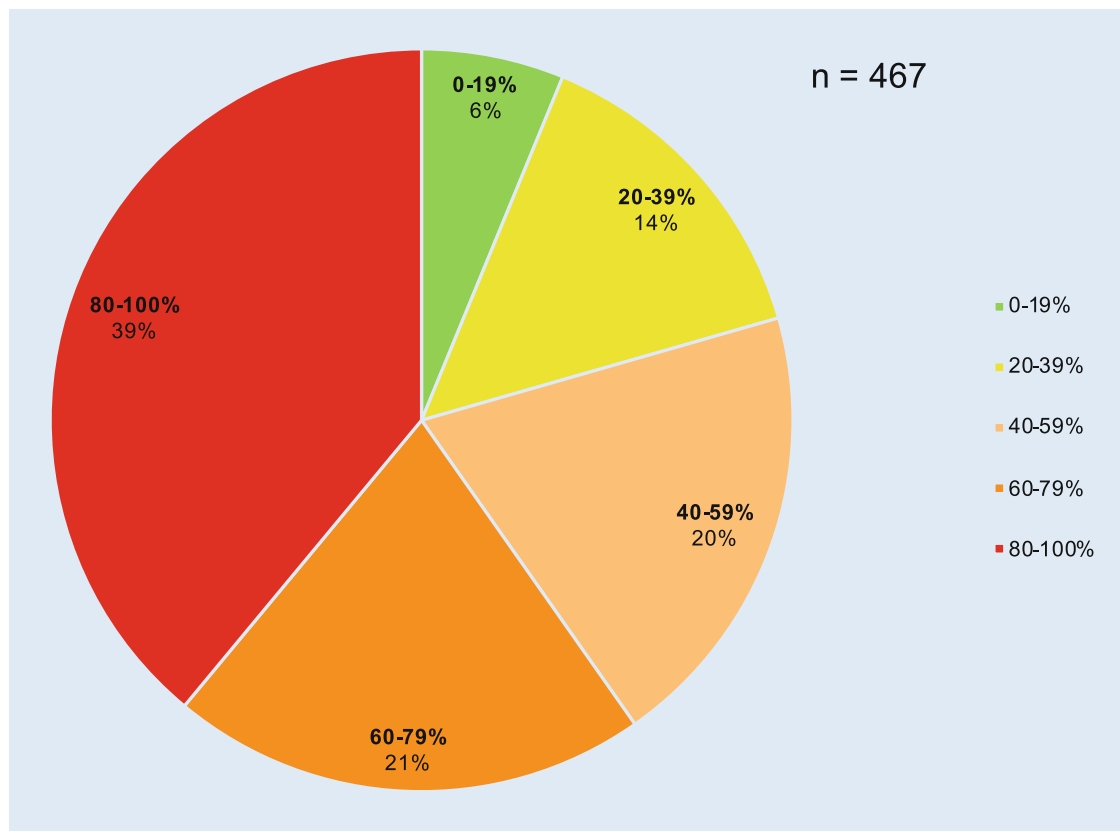

Fig. $3 \Delta$ Distribution of the extent of the reduction in surgical capacity $(n=467)$. In a total of $60.0 \%$ of cases, there was a reduction in surgical capacity from $60.0 \%$ to $100 \%$. Only $6.0 \%$ reported a reduction of less than $20.0 \%$

at $-34.9 \%$, perforating eyeball injuries ( 51 vs. 55$)$ at $-7.3 \%$, acute glaucoma (198 vs. $242)$ at $-17.8 \%$, CRAO and AION (256 vs. 371 ) at $-31.0 \%$, and "others" (1027 vs. 1487 ) at a reduced number of $-31.0 \%$. The number of cases for penetrating keratoplasty and amniotic membrane transplantation (180 vs. 440) also decreased significantly at $-59.1 \%$, whereas an increase of $+8.2 \%$ (66 vs. 61 ) was recorded for the diagnosis of endophthalmitis.

\section{Reduction in inpatient ward capacities}

Overall, $77.3 \%$ of all facilities with inpatient wards were affected by a loss of capacities, either by a reduction in the number of beds $(35.2 \%)$ or ward closures $(42.1 \%)$. Capacity reductions affected clinics $(80.0 \%)$ more often than inpatient wards $(73.0 \%)$, but these consisted more often of ward closures in inpatient wards $(50.0 \%)$ than in clinics (37.0\%; - Fig. 7). Departments affected by reductions of inpatient capacities or ward closures gave up $78.5 \%$ of their beds on average. Beds in inpatient wards were reduced on average by $88.3 \%$, and in clinics by $72.6 \%$.

\section{Staff shortages and reasons for restrictions on care}

A total of $76.0 \%$ of survey participants stated that legal, institutional, or professional policy requirements had led to restrictions on ophthalmic care. Operational or physical restrictions were mentioned in $15.5 \%$, "avoidance of risk to own health" was cited as the most frequent reason in $39.0 \%$, and appointment cancellations by patients in $84.0 \%$ (• Fig. 8 ). A total of $25.0 \%$ of respondents stated that they had experienced staff shortages due to SARS-CoV-2 infections or quarantine measures. Participants at clinics with inpatient beds also reported having reassigned specialists (2.9\%), assistant physicians $(6.3 \%)$, nursing staff $(5.9 \%)$, and medical assistants (5.6\%) to other departments.

\section{Discussion}

The results of the survey conducted by the commission for cross-sectoral ophthalmology of the German Ophthalmological Society (DOG) and the Professional Association of German Ophthalmologists (BVA) provide the first overall impression of the occasionally dramatic changes in ophthalmic care in Germany during the first weeks of the SARS-CoV-2 pandemic. This resulted in considerable restrictions on the performance of elective treatments and procedures worldwide and across disciplines due to the conversion of hospitals to deal with the expected care of COVID-19 patients and the provision of the largest possible intensive care bed capacities, as well as the implementation of social distancing and hygiene rules [1-12].

The present results of an evaluation of a total of 1190 participants show that all sectors of ophthalmology, both outpatient and inpatient care, were affected in this historically unprecedented and exceptional medical situation, with only few exceptions. Thus, only $5.8 \%$ of respondents stated that they had carried out their outpatient or consultation activities without restrictions in the period from March 15 to April 15, 2020. In contrast, $63.5 \%$ of participants responded that they had maintained reduced treatment during this period, and $27.5 \%$ emergency treatment only. The treatment of patients apparently continued to be provided in an unrestricted manner, since only $2.4 \%$ indicated a reduced intensity of treatment, e.g., by telephone consultations only [15, 16]. As a result, practices were permanently available for treatment rather than being closed. Regardless of the type of medical institution, conservative treatment of patients was reduced in 89.0 to $98.0 \%$ of cases due to the pandemic-related "lockdown" or limited to the treatment of emergencies, i.e., regardless of whether it was a private outpatient practice or an inpatient clinic. In contrast, only 2.0 to $9.0 \%$ provided unrestricted outpatient or consultation hours.

Overall, ophthalmic care providers have contributed their share to overcoming the pandemic-related crisis in Germany and yet, at the same time, have been able to maintain care focused on emergency and necessary treatments within the shortest possible time. Thus, the trend in ophthalmic care in the first weeks of the pandemic largely reflects the development of care strategies in other disciplines [4-12]. However, the current data also demonstrate that one focus of the measures taken to overcome 
Outpatient surgery in a private practice

$(n=49)$

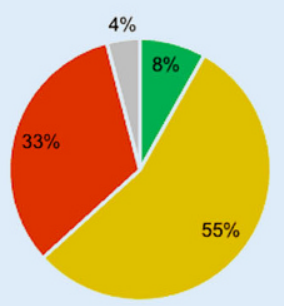

- Unrestricted care (4)

- Reduced care (27)

- Emergency care only (16)

- Not applicable (2)
Outpatient surgery in an MCC/group practice $(n=110)$

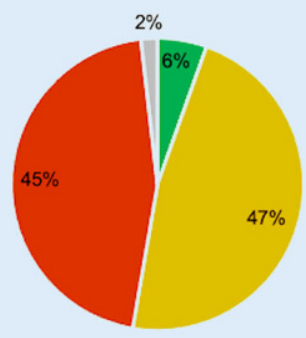

- Unrestricted care (6)

- Reduced care (52)

- Emergency care only (50)

- Not applicable (2)

\section{Outpatient surgery on inpatient wards}

$(n=12)$

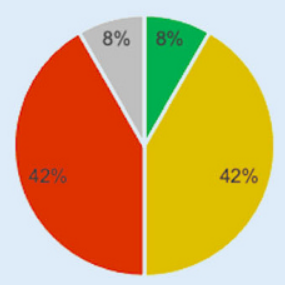

- Unrestricted care (1)

" Reduced care (5)

- Emergency care only (5)

- Not applicable (1)
Inpatient surgery on inpatient wards $(n=12)$

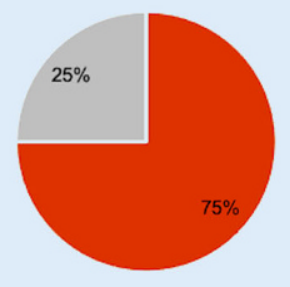

- Unrestricted care (0)

- Reduced care (0)

- Emergency care only (9)

- Not applicable (3)
Outpatient surgery in clinics

$(n=34)$

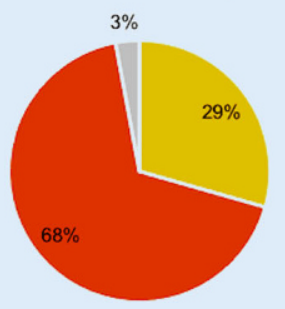

- Unrestricted care (0)

- Reduced care (10)

- Emergency care only (23)

- Not applicable (1)
Inpatient surgery in clinics

$(n=34)$

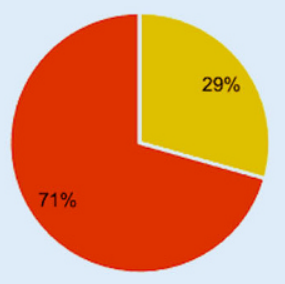

- Unrestricted care (0)

- Reduced care (10)

- Emergency care only (24)

- Not applicable (0)

Fig. $4 \Delta$ Restriction of surgical activities related to the type of facility. The performance of inpatient procedures was limited to emergency care only in $75.0 \%$ of inpatient wards and in $71.0 \%$ of clinics, as was outpatient surgery in $68.0 \%$ of clinics and in $42.0 \%$ of inpatient wards, while this proportion was $45.0 \%$ in surgical medical care centers (MCCS) and group practices and $33.0 \%$ in private surgical practices

the crisis was to provide inpatient bed and treatment capacities. In particular, inpatient procedures during the pandemic were largely limited to emergency care. This was the case in $75.0 \%$ of inpatient wards and $71.0 \%$ of clinics. In addition, $68.0 \%$ of clinics and $42.0 \%$ of inpatient wards provided outpatient surgery exclusively for the surgical treatment of emergencies, whereas this was the case in only $33.0 \%$ of private surgical practices.

This difference can be explained by the fact that most hospitals have a very consistent strategy for responding to the challenges of the COVID-19 pandemic by focusing exclusively on emergency and urgent care, thereby ensuring a continu- ous capacity of intensive care and monitoring beds required for the treatment of COVID 19 patients [1-3, 8-10]. In particular, inpatient surgery became a scarce resource, which is also reflected in the reduction in bed capacity. A total of $77.3 \%$ of all facilities with inpatient beds were affected by a reduction in the number of beds or ward closures, characterized by an average loss of $78.5 \%$ of their beds. This effect was further exacerbated by the deployment of ophthalmic personnel to infectious disease outpatient clinics set up in many places at the beginning of the pandemic to maintain COVID19 treatment capacity, as well as the frequent practice of splitting medical teams in hospitals with inpatient beds to ensure a reserve of personnel. In addition, inpatient clinics also reported transferring $2.8 \%$ of their specialists, $6.3 \%$ of their residents, $5.9 \%$ of their nurses, and $5.6 \%$ of their medical assistants to other specialty areas.

A comparison of the case numbers for penetrating keratoplasty and amniotic membrane transplantation for the periods from January 15 to February 15, 2020, and March 15 to April 15, 2020 [1], shows that the considerable reduction of inpatient capacities in ophthalmology also had an effect on the performance of procedures that were classified as urgent according to the crisis plan for basic, non-elective ophthalmic care. Accordingly, there was a significant decrease of 


\section{Originalien}

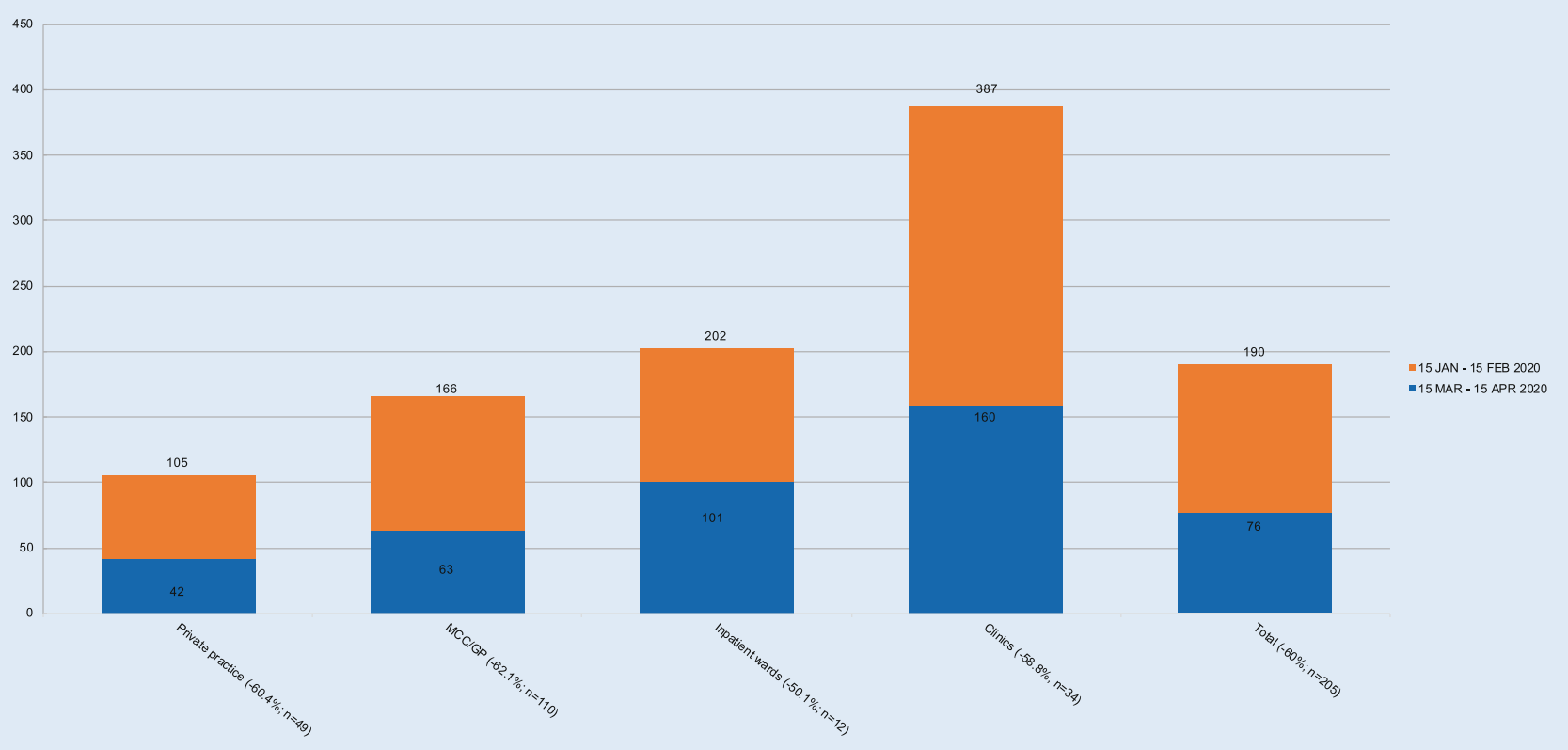

Fig. $5 \Delta$ Trend in the number of surgeries in the period from March 15, 2020, to April 15, 2020, compared to January 15, 2020, to February $15,2020(n=205)$. While the absolute number of reduced surgeries in relation to the type of facility was highest in clinics, the relative share was $58.8 \%$, which corresponds to an average for all facilities of $60.0 \%$.MCC medical care center, GPgroup practices

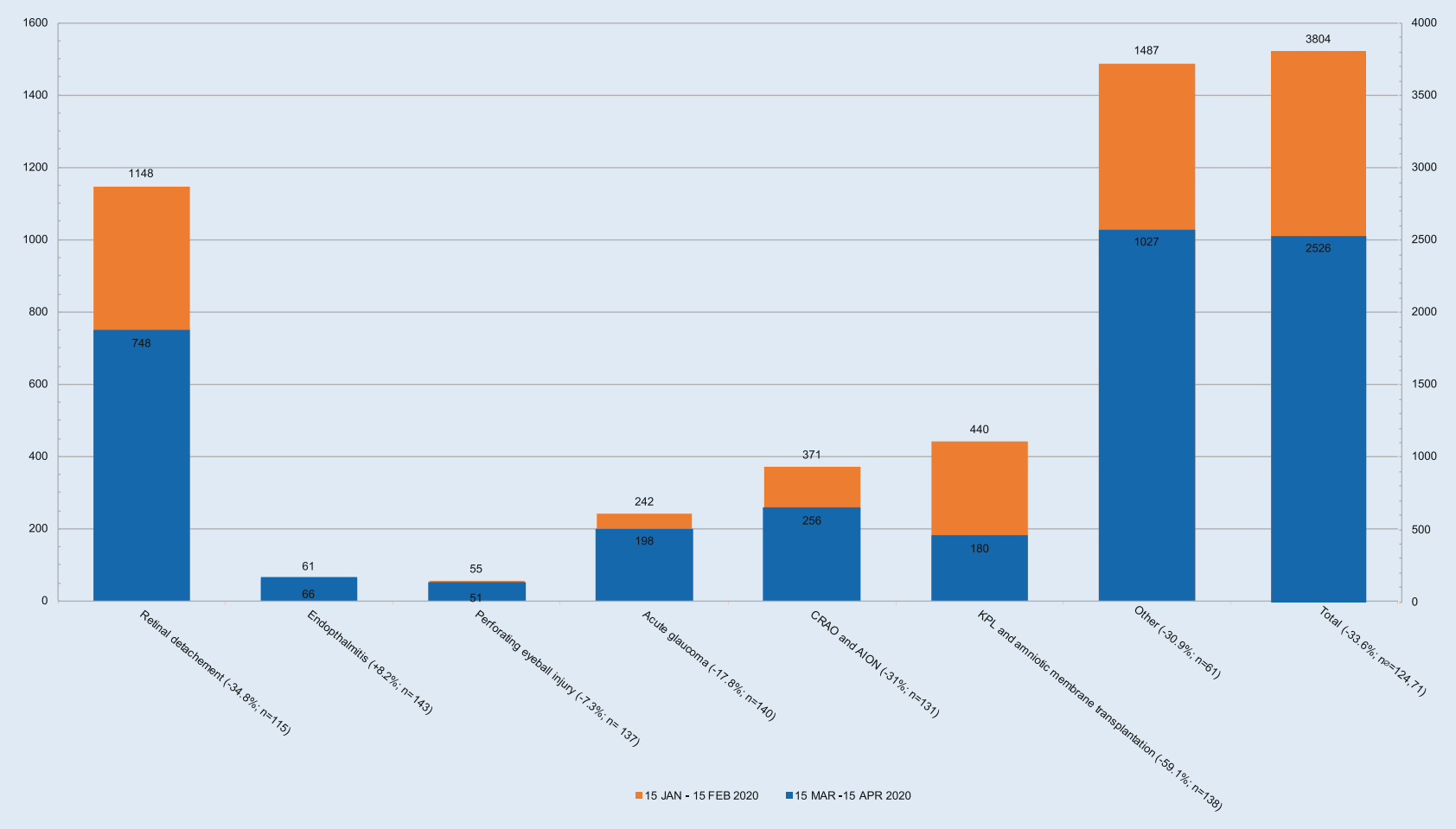

Fig. $6 \Delta$ Comparison of the case numbers for emergencies and urgent procedures between January 15 to February 15, 2020, and March 15 to April 15,2020. With a total number of 2526 compared to 3804 cases, there was a significant decrease of $-34.0 \%$. The largest decreases were for retinal detachment ( $748 \mathrm{vs.} 1148)$ at $-34.8 \%$, central retinal artery occlusion (CRAO) and anterior ischemic optic neuropathy ( $A I O N ; 256$ vs. 371 ) at $-31.0 \%$. There was also a significant decrease in the number of cases of penetrating keratoplasty (KPL) and amniotic membrane transplantation ( $180 \mathrm{vs.440)}$ at $-59.1 \%$. The only increase was reported for endophthalmitis at $+8.2 \%$ 


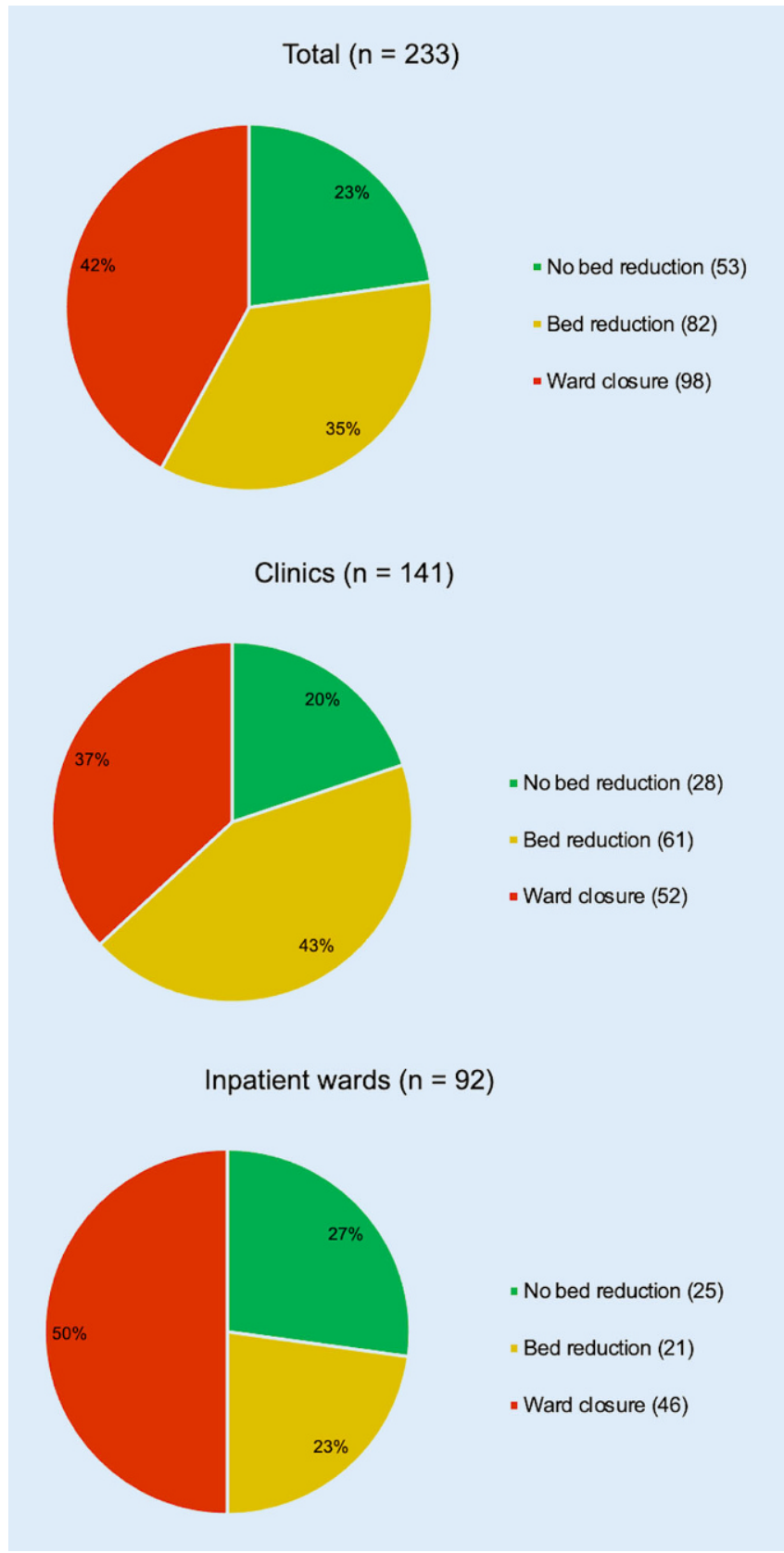

Fig. $7<$ A total of $77.3 \%$ of all facilities with inpatient beds were affected by a reduction of inpatient capacities, in particular inpatient wards (50.0\%) by ward closures
$-59.1 \%$ in the number of interventions for these indications during the initial phase of the pandemic.

A comparison of case numbers for the emergency diagnoses retinal detachment, endophthalmitis, perforating eyeball injury, acute glaucoma, central artery occlusion and anterior ischemic optic neuropathy in the period from March 15 to April 15, 2020, vs. January 15 to February 15,2020 , also showed a significant decrease of $34.0 \%$. Particularly striking are the significantly lower treatment numbers for retinal detachment $(-34.8 \%)$ and thalmic care. On the other hand, based on the current data, one cannot rule out that there was a change in patient flow during the pandemic, as the survey only covered a proportion of all ophthalmic facilities in Germany. Therefore, a direct comparison must be interpreted with caution. However, since retinal detachment is a disease that is not necessarily immediately noticed by affected patients, often being detected only by fundoscopy, one can assume that in some cases during the initial phase of the pandemic, patients failed to present or presented late to the ophthalmologist due to the absence of symptoms.

Similar trends during the pandemic have also been reported in other medical disciplines. For example, numerous publications now indicate that the number of cardiovascular emergencies such as myocardial infarction or stroke decreased during the pandemic, in some cases significantly [17-20]. It is assumed that this is not only due to delayed diagnosis due to limited treatment services, but possibly also to a protective effect of lifestyle changes (stress reduction through home office, avoidance of business trips, etc.) [20]. Thus, secondary effects of the pandemic may also have contributed to a reduction in the number of cases of CRAO and AION, for example.

Another reason for the decrease in the number of emergencies observed across disciplines during the initial phase of the pandemic is assumed to be patients' avoidance behavior due to the fear of infection ("coronavirus angst") in medical facilities $[13,14]$. Indeed, $84.0 \%$ of survey participants stated that appointment cancellations by patients had led to a reduction in ophthalmic care, and as many as $39.0 \%$ reported avoidance of risk to own health as a reason. In fact, $25.0 \%$ of survey participants stated that they had experienced staff shortages due to SARS-CoV-2 infections or quarantine measures. In the meantime, however, the risks of potential infection have been minimized by the knowledge gained during the first months of the pandemic and the widespread introduction of additional hygiene measures (face masks, plastic shields on slit lamps) [1, 21-36]. 


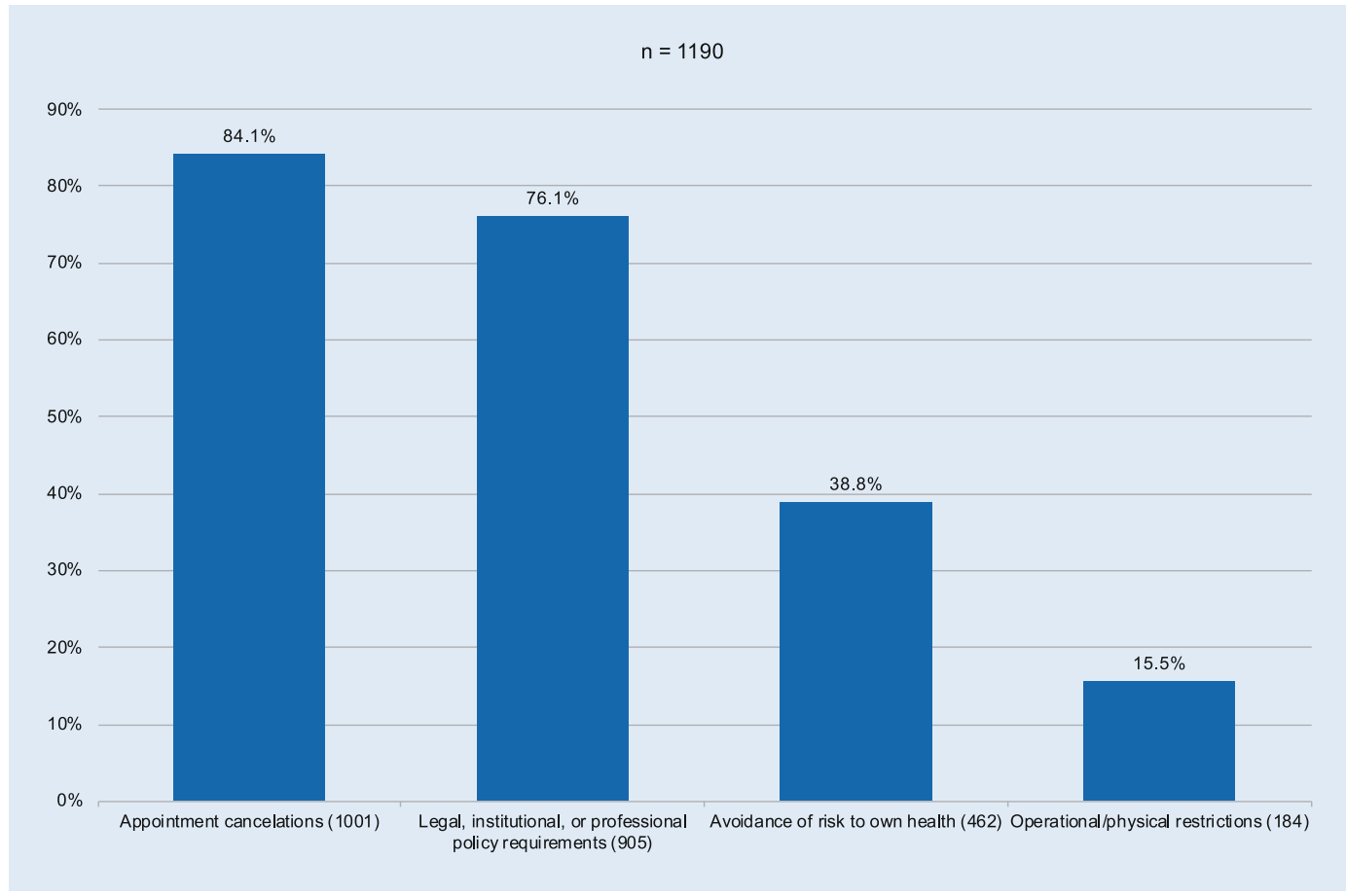

Fig. $8<$ Reasons for restricting treatment. Most frequently, legal, institutional, or professional policy requirements were cited as reasons for restrictions on ophthalmic care
Contrary to the trend in the aforementioned diagnoses, only a slight decrease was recorded for perforating eyeball injuries at $-7.3 \%$ and an increase for endophthalmitis at $+8.2 \%$. While the incidence of perforating eyeball injuries can be explained by the general "lockdown," and both rare events by the range of fluctuation within the short observation periods, the consistently slightly increased number of endophthalmitis cases possibly also corresponds to the generally maintained surgical treatment and simultaneously increased severity of cases. At most facilities, the continuation of intravitreal injections, which are bound to a fixed treatment plan, was still possible to a partially reduced extent while complying with hygiene measures $[1,37]$. However, there is a possible association between endophthalmitis risk and changes in behavior, e.g., excessive wearing of face masks by patients, which could have directly or indirectly led to an increased exposure to nasopharyngeal bacteria [38].

\section{Conclusion}

The analysis of the present survey conducted by the German commission for cross-sectoral ophthalmology, a joint commission of the DOG and the
BVA, provides the first comprehensive overview of the effects of the SARSCoV-2 pandemic on ophthalmic care in Germany. According to this survey, there was a massive reduction in non-urgent outpatient and inpatient as well as conservative and surgical ophthalmic care during the initial phase of the pandemic from March 15 to April 15, 2020, which affected all areas of ophthalmology.

Although the general medical "lockdown" focused on provisioning inpatient and intensive care capacities, which in particular reduced the provision of inpatient treatment exclusively to emergency care, the implementation of measures to maintain ophthalmic patient care was characterized by a considerable crosssectoral solidarity.

During the initial phase of the pandemic, emergency care and the treatment of urgent cases were maintained despite extensive restrictions imposed by legal, institutional, or professional policies, as well as the implementation of the necessary hygiene measures. At any time during this phase of the pandemic, primary outpatient care and continuous inpatient care were ensured by the facilities that remained open in both sectors.

However, similar to reports from other disciplines, a significant decrease in case numbers was observed, which can be explained in part by the generally reduced scope of medical care, as well as by secondary effects of the pandemic. The current data demonstrate not only the importance of ophthalmic care, but also its adaptability and significance during the crisis, from which ophthalmology will emerge stronger for future challenges.

\section{Corresponding address}

Prof. Dr. Lars-Olof Hattenbach, F.E.B.O.

Eye Clinic, Ludwigshafen Hospital

Bremserstr. 79, 67063 Ludwigshafen, Germany hattenbach.LO@klilu.de

hattenbach@me.com

\section{Declarations}

Conflict of interest. L.-O. Hattenbach, P. Heinz, N. Feltgen, H. Hoerauf, T. Kohnen, S. Priglinger, W. Bachmann, J. Rieks, N. Eter, and T. Reinhard declare that they have no competing interests.

For this paper the authors did not perform any studies in humans or animals. The ethical guidelines for the studies listed apply to the respective studies.

The supplement containing this article is not sponsored by industry. 


\section{References}

1. Hattenbach LO, Reinhard T, Walter P et al (2020) Crisis management strategies of hospitals during the pandemic. Ophthalmologe 117(7):652-658. https://doi.org/10.1007/s00347-020-01162-x

2. Poeran J,Zhong H, Wilson Letal (2020) Cancellation of elective surgery and intensive care unit capacity in New York state: a retrospective cohort analysis. Anesth Analg. https://doi.org/10.1213/ANE. 0000000000005083

3. Tonna JE, Hanson HA, Cohan JN et al (2020) Balancing revenue generation with capacity generation: case distribution, financial impact and hospital capacity changes from cancelling or resuming elective surgeries in the US during COVID-19. medRxiv. https://doi.org/10.1101/ 2020.04.29.20066506

4. Ralli M, Minni A, Candelori F et al (2020) Effects of COVID-19 pandemic on otolaryngology surgery in Italy: the experience of our university hospital. Otolaryngol Head Neck Surg. https://doi.org/10. 1177/0194599820928970

5. Taha MA, Hall CA, Rathbone RF et al (2020) Rhinologic procedures in the era of COVID19: health-care provider protection protocol. Am J Rhinol Allergy. https://doi.org/10.1177/ 1945892420927178

6. Gupta A, Arora V, Nair D et al (2020) Status and strategies for the management of head and neck cancer during COVID-19 pandemic: Indian scenario. Head Neck. https://doi.org/10.1002/hed. 26227

7. Boettler T, Newsome PN, Mondelli MU (2020) Care of patients with liver disease during the COVID-19 pandemic: EASL-ESCMID position paper. JHEP Rep 2(3):100113

8. CovidSurg Collaborative, Nepogodiev D, Bhangu A (2020) Elective surgery cancellations Due to the COVID-19 pandemic: global predictive modelling to inform surgical recovery plans. Br JSurg. https:// doi.org/10.1002/bjs.11746

9. Argenziano M, FischkoffK, Smith CR (2020) Surgery scheduling in a crisis. NEngl J Med 382:e87

10. Diaz A, Sarac BA, Schoenbrunner AR et al (2020) Elective surgery in the time of COVID-19. Am JSurg. https://doi.org/10.1016/j.amjsurg.2020.04.014

11. Karampelias V, Spanidis Y, Kehagias I (2020) Surgical practice and operative surgical strategies during the COVID-19T pandemic: a commentary. Ann Med Surg 55:47-48

12. Skalet $A H$, Allen $R C$, Shields $C L$ et al (2020) Considerations for the management and triage of ocular oncology cases during the COVID-19 pandemic. Ocul Oncol Pathol 6(3):1-4

13. Lazzerini M, Barbi E, Apicella A (2020) Delayed access or provision of care in Italy resulting from fear of COVID-19. Lancet Child Adolesc Health 4(5):e10-e11

14. Yıldırım M, Geçer E, Akgül Ö et al (2020) The impacts of vulnerability, perceived risk, and fear on preventive behaviours against COVID-19. Psychol Health Med 3:1-9

15. Saleem SM, Pasquale LR, Sidoti PA et al (2020) Virtual ophthalmology: telemedicine in a Covid-19 era. Am J Ophthalmol. https://doi.org/10.1016/j. ajo.2020.04.029

16. Wasser LM, Assayag E, Tsessler M et al (2020) Response of ophthalmologists in Israel to the novel coronavirus (2019-nCoV) outbreak. Graefes Arch Clin Exp Ophthalmol 28:1-8

17. Holt A, Gislason GH, Schou M et al (2020) Newonset atrial fibrillation: incidence, characteristics, and related events following a national COVID-19 lockdown of 5.6 million people. Eur Heart J. https:// doi.org/10.1093/eurheartj/ehaa494

18. De Rosa S, Spaccarotella C, Basso C et al (2020) Reduction of hospitalizations for myocardial infarction in Italy in the COVID-19 era. Eur Heart J 41(22):2083-2088. https://doi.org/10. 1093/eurheartj/ehaa409

19. Lange SJ, Ritchey MD, Goodman $A B$ et al (2020) Potential indirect effects of the COVID-19 pandemic on use of emergency departments for acute life-threatening conditions - United States, January-May 2020. MMWR Morb Mortal Wkly 69(25):795-800

20. Zitelny E, Newman N, Zhao D (2020) STEM during the COVID-19 pandemic - an evaluation of incidence. Cardiovasc Pathol 48:107232

21. Arons MM, Hatfield KM, Reddy SC (2020) Presymptomatic SARS-coV-2 infections and transmission in a skilled nursing facility. $\mathrm{N}$ Engl J Med 382:2081-2090

22. Glauser W (2020) Proposed protocol to keep COVID-19 out of hospitals. CMAJ 192(10):E264-E265

23. Olivia Li JP, Shantha J, Wong TY (2020) Preparedness among ophthalmologists: during and beyond the COVID-19 pandemic. Ophthalmology 127(5):569-572

24. Xia J, Tong J, Liu M et al (2020) Evaluation of coronavirus in tears and conjunctival secretions of patients with SARS-CoV-2 infection. J Med Virol 92:589-594

25. Seah IYJ, Anderson DE, Kang AEZ et al (2020) Assessing viral shedding and infectivity of tears in coronavirus disease 2019 (COVID-19) patients. Ophthalmology. https://doi.org/10. 1016/j.ophtha.2020.03.026

26. Guan WJ, Ni ZY, Hu Y, Liang WH et al (2020) Clinical characteristics of coronavirus disease 2019 in China. NEngl J Med 382(18):1708-1720

27. Lange C, Wolf J, Auw-Haedrich C et al (2020) Expression of the COVID-19 receptor ACE2 in the human conjunctiva. J Med Virol. https://doi.org/ 10.1002/jmv.25981

28. Lim LW, Yip LW, Tay HW et al (2020) Sustainable practice of ophthalmology during COVID-19: challenges and solutions. Graefes Arch Clin Exp Ophthalmol 21:1-10

29. Breazzano MP, Shen J, Abdelhakim AH et al (2020) New York city COVID-19 resident physician exposure during exponential phase of pandemic JClin Invest. https://doi.org/10.1172/JCI139587

30. Van Gerven L, Hellings PW, Cox T et al (2020) Personal protection and delivery of rhinologic and endoscopic skull base procedures during the COVID-19 outbreak. Rhinology 58(3):289-294

31. Arrigo A, Aragona E, Parodi MB et al (2020) Ophthalmology and SARS-coV-2: blind toward those who fight blindness? Eur J Ophthalmol. https://doi.org/10.1177/1120672120929961

32. Loon SC, Teoh SC, Oon LL et al (2004) The severe acute respiratory syndrome coronavirus in tears. $\mathrm{Br}$ JOphthalmol 88(7):861-863

33. Heiduschka $\mathrm{P}$, Lauermann JL, Bosche FD, Eter $\mathrm{N}$ (2020) (2020). Ein Schutz gegen die Tröpfcheninfektion an der Spaltlampe. Sicherheit für die Augenärzte UND die Patienten. Ophthalmologe 117(4):296-297

34. Lange C, Wolf J, Auw-Hädrich C et al (2020) Welche Bedeutung hat die Bindehaut als möglicher Übertragungsweg für eine SARS-Cov-2-Infektion? Ophthalmologe 117:626-630

35. Wacker K, Reinhard T (2020) Spezielle ophthalmologische Schutzmaßnahmen in der COVID-19Pandemie. Ophthalmologe 117:638-641
36. Singh P, Müller M, Hack D et al (2020) Entwicklung und Implementierung eines Betriebs-konzeptes in einer Universitätsaugenklinik im Rahmen der SARS-CoV-2-Pandemie. Ophthalmologe 117:595-601

37. Korobelnik JF, Loewenstein A, Eldem B et al (2020) Guidance for anti-VEGF intravitreal injections during the COVID-19 pandemic. Graefes Arch Clin Exp Ophthalmol 258:1149-1156

38. Wen JC, McCannel CA, Mochon AB, Garner OB (2020) Bacterial dispersal associated with speech in the setting of intravitreous injections. Arch Ophthalmol 129(12):1551-1554 